\title{
The Ellipse Law: Kirchhoff Meets Dislocations
}

\author{
J. A. Carrillo ${ }^{1}$, J. Mateu ${ }^{2}$, M. G. Mora ${ }^{3}$, L. Rondi ${ }^{4}$, L. Scardia ${ }^{5}$,
} J. Verdera ${ }^{2}$

${ }^{1}$ Department of Mathematics, Imperial College London, London, United Kingdom.

E-mail: carrillo@imperial.ac.uk

2 Department de Matemàtiques, Universitat Autònoma de Barcelona, Catalonia, Spain.

E-mail: mateu@mat.uab.cat; jvm@mat.uab.cat

3 Dipartimento di Matematica, Università di Pavia, Pavia, Italy.

E-mail: mariagiovanna.mora@unipv.it

4 Dipartimento di Matematica, Università di Milano, Milano, Italy.

E-mail: luca.rondi@unimi.it

5 Department of Mathematics, Heriot-Watt University, Edinburgh, United Kingdom.

E-mail: L.Scardia@hw.ac.uk

Received: 17 December 2018 / Accepted: 5 January 2019

Published online: 24 April 2019 - (C) The Author(s) 2019

\begin{abstract}
In this paper we consider a nonlocal energy $I_{\alpha}$ whose kernel is obtained by adding to the Coulomb potential an anisotropic term weighted by a parameter $\alpha \in \mathbb{R}$. The case $\alpha=0$ corresponds to purely logarithmic interactions, minimised by the circle law; $\alpha=1$ corresponds to the energy of interacting dislocations, minimised by the semicircle law. We show that for $\alpha \in(0,1)$ the minimiser is the normalised characteristic function of the domain enclosed by the ellipse of semi-axes $\sqrt{1-\alpha}$ and $\sqrt{1+\alpha}$. This result is one of the very few examples where the minimiser of a nonlocal anisotropic energy is explicitly computed. For the proof we borrow techniques from fluid dynamics, in particular those related to Kirchhoff's celebrated result that domains enclosed by ellipses are rotating vortex patches, called Kirchhoff ellipses.
\end{abstract}

\section{Introduction}

The starting point of our analysis is the nonlocal energy

$$
I_{\alpha}(\mu)=\frac{1}{2} \iint_{\mathbb{R}^{2} \times \mathbb{R}^{2}} W_{\alpha}(x-y) d \mu(x) d \mu(y)+\frac{1}{2} \int_{\mathbb{R}^{2}}|x|^{2} d \mu(x)
$$

defined on probability measures $\mu \in \mathcal{P}\left(\mathbb{R}^{2}\right)$, where the interaction potential $W_{\alpha}$ is given by

$$
W_{\alpha}\left(x_{1}, x_{2}\right)=-\frac{1}{2} \log \left(x_{1}^{2}+x_{2}^{2}\right)+\alpha \frac{x_{1}^{2}}{x_{1}^{2}+x_{2}^{2}}, \quad x=\left(x_{1}, x_{2}\right) \in \mathbb{R}^{2},
$$

and $\alpha \in \mathbb{R}$. Here the parameter $\alpha$ has the role of tuning the strength of the anisotropic component of $W_{\alpha}$, making it more or less prominent.

In the particular case where the anisotropy is switched off, namely for $\alpha=0$, the minimiser is radial, and is given by the celebrated circle law $\mu_{0}:=\frac{1}{\pi} \chi_{B_{1}(0)}$, the normalised characteristic function of the unit disc. This result is now classical and has been 
proved in a variety of contexts, from Fekete sets to orthogonal polynomials, from random matrices to Ginzburg-Landau vortices and Coulomb gases (see, e.g., [26,39], and the references therein).

In the case $\alpha=1$, the energy $I_{1}$ models interactions between edge dislocations of the same sign (see, e.g., $[27,36]$ ). The minimisers of $I_{1}$ were since long conjectured to be vertical walls of dislocations, and this has been confirmed only very recently, in [37], where the authors proved that the only minimiser of $I_{1}$ is the semi-circle law

$$
\mu_{1}:=\frac{1}{\pi} \delta_{0} \otimes \sqrt{2-x_{2}^{2}} \mathcal{H}^{1}\llcorner(-\sqrt{2}, \sqrt{2})
$$

on the vertical axis.

In this paper we explicitly characterise the minimiser of $I_{\alpha}$ for every $\alpha \in \mathbb{R}$. It turns out that the values $\alpha= \pm 1$ are critical values of the parameter, corresponding to maximal anisotropy, at which an abrupt change in the dimension of the support of the minimiser occurs. Indeed, for $\alpha \in(-1,1)$ we prove that the unique minimiser of $I_{\alpha}$ is the normalised characteristic function of the region surrounded by an ellipse of semi-axes $\sqrt{1-\alpha}$ and $\sqrt{1+\alpha}$. On the other hand, we show that for every $\alpha \geq 1$ the only minimiser of $I_{\alpha}$ is the semi-circle law $\mu_{1}$ on the vertical axis, while for $\alpha \leq-1$ it is the semi-circle law on the horizontal axis.

Minimisers of nonlocal energies have been explicitly determined only in very few cases. Even in the classical case of purely logarithmic interactions, the characterisation of the equilibrium measure for a general confinement is still an open problem, and is the object of an intense research activity (see $[4,8,9,26,32]$ for the study of various confinements of special form). For anisotropic interaction kernels with logarithmic singularity, the present contribution, together with [37], is the only result of explicit computation of the equilibrium measure.

The main result of the paper is the following:

Theorem 1.1. Let $0 \leq \alpha<1$. The measure

$$
\mu_{\alpha}:=\frac{1}{\sqrt{1-\alpha^{2}} \pi} \chi_{\Omega(\sqrt{1-\alpha}, \sqrt{1+\alpha})},
$$

where

$$
\Omega(\sqrt{1-\alpha}, \sqrt{1+\alpha}):=\left\{x=\left(x_{1}, x_{2}\right) \in \mathbb{R}^{2}: \frac{x_{1}^{2}}{1-\alpha}+\frac{x_{2}^{2}}{1+\alpha}<1\right\},
$$

is the unique minimiser of the functional $I_{\alpha}$ among probability measures $\mathcal{P}\left(\mathbb{R}^{2}\right)$, and satisfies the Euler-Lagrange conditions

$$
\begin{aligned}
& \left(W_{\alpha} * \mu_{\alpha}\right)(x)+\frac{|x|^{2}}{2}=C_{\alpha} \text { for every } x \in \Omega(\sqrt{1-\alpha}, \sqrt{1+\alpha}), \\
& \left(W_{\alpha} * \mu_{\alpha}\right)(x)+\frac{|x|^{2}}{2} \geq C_{\alpha} \text { for every } x \in \mathbb{R}^{2},
\end{aligned}
$$

with

$$
\begin{aligned}
C_{\alpha} & =2 I_{\alpha}\left(\mu_{\alpha}\right)-\frac{1}{2} \int_{\mathbb{R}^{2}}|x|^{2} d \mu_{\alpha}(x) \\
& =\frac{1}{2}-\log \left(\frac{\sqrt{1-\alpha}+\sqrt{1+\alpha}}{2}\right)+\alpha \frac{\sqrt{1-\alpha}}{\sqrt{1-\alpha}+\sqrt{1+\alpha}} .
\end{aligned}
$$


The result for $\alpha>1$ can be obtained by a simple comparison argument, and for $\alpha<0$ by symmetry (see Sect. 2). We emphasise that for $0 \leq \alpha \leq 1$ the Euler-Lagrange conditions (1.5)-(1.6) are a sufficient condition to minimality, since we will show that the energy $I_{\alpha}$ is strictly convex for these values of $\alpha$ (see Proposition 2.1). Thus, proving that $\mu_{\alpha}$ satisfies (1.5)-(1.6) immediately entails the minimality of $\mu_{\alpha}$.

1.1. Kirchhoff ellipses and dislocations. To prove that the ellipse law $\mu_{\alpha}$ satisfies the Euler-Lagrange conditions (1.5)-(1.6), we evaluate the convolution of the kernel $W_{\alpha}$ with the characteristic function of the domain enclosed by a general ellipse. Let us define, for any $a, b>0$, the domain

$$
\Omega(a, b):=\left\{x=\left(x_{1}, x_{2}\right) \in \mathbb{R}^{2}: \frac{x_{1}^{2}}{a^{2}}+\frac{x_{2}^{2}}{b^{2}}<1\right\},
$$

which is the region surrounded by an ellipse centred at the origin with horizontal semiaxis $a$ and vertical semi-axis $b$. As a first step, we compute explicitly the gradient of $W_{\alpha} * \chi_{\Omega(a, b)}$, both inside and outside $\Omega(a, b)$; see Eqs. (3.6)-(3.7) in Proposition 3.1. As we shall see, this is enough to conclude the proof of Theorem 1.1, but for completeness we shall also explicitly compute $W_{\alpha} * \chi_{\Omega(a, b)}$ in the whole plane (see Remark 4.1). The gradient of $W_{\alpha} * \chi_{\Omega(a, b)}$ is the sum of $-(1 / z) * \chi_{\Omega(a, b)}$, where $z=x_{1}+i x_{2}$ is the complex variable in the plane, and of a second term containing the gradient of the anisotropic part of the potential. The convolution $-(1 / z) * \chi_{\Omega(a, b)}$ has been computed before, for instance in [28], for rotating vortex patches in fluid dynamics.

Let us recall that a vortex patch is the solution of the vorticity form of the planar Euler equations in which the initial condition is the characteristic function of a bounded domain $D_{0}$. Since vorticity is transported by the flow, the vorticity at time $t$ is the characteristic function of a domain $D_{t}$. In general the evolution of $D_{t}$ is an extremely complicated phenomenon, but Kirchhoff proved more than one century ago that if $D_{0}$ is the domain enclosed by an ellipse with semi-axes $a$ and $b$, then $D_{t}$ is just a rotation of $D_{0}$ around its centre of mass with constant angular velocity $\omega=a b /(a+b)$, see [25,30,34]. Domains with the simple evolution property described above are called $V$-states or rotating vortex patches. They can be viewed as stationary solutions in a reference system that rotates with the patch, and they can be described by means of an equation involving the stream function $-\log |\cdot| * \chi_{D_{0}}$ of the initial patch $D_{0}$ (see [11]), which is formally similar to the Euler-Lagrange equation (1.5). If one wants to verify that for the elliptical patch $\Omega(a, b)$ such equation is satisfied, one needs to compute explicitly $-\log |\cdot| * \chi_{\Omega(a, b)}$, and this can be done by first computing its gradient $-(1 / z) * \chi_{\Omega(a, b)}$.

This is the main connection between Kirchhoff ellipses and dislocations. However, the challenge in the dislocations case is to compute the gradient of the anisotropic part of $W_{\alpha} * \chi_{\Omega(a, b)}$. The key observation is that it can be written in terms of suitable complex derivatives of the fundamental solution of the operator $\partial^{2}$, where $\partial=\partial / \partial z$. To compute such term explicitly we need the expression of $-(1 / z) * \chi_{\Omega(a, b)}$, which was known, as well as the expression of $\left(z / \bar{z}^{2}\right) * \chi_{\Omega}(a, b)$, which we obtain in Proposition 3.1.

What is surprising is that techniques developed in the context of fluid mechanics turn out to be crucial for the characterisation of the minimisers of the anisotropic energy $I_{\alpha}$, which arises, in the case $\alpha=1$, in the context of edge dislocations in metals. In particular the minimality of the semi-circle law for the dislocation energy $I_{1}$ can be deduced from Theorem 1.1 by a limiting argument based on $\Gamma$-convergence (see Corollary 3.3 ). That 
is, we obtain again the main result of [37], but with a different proof based on methods from fluid mechanics and complex analysis.

It is worth emphasising the special role that ellipses play in both contexts. On the one hand, in fluid mechanics they provide one of the few explicit solutions of the incompressible Euler equations. On the other hand, the characteristic function of the elliptical domains $\Omega(a, b)$ is one of the few measures $\mu$ for which the convolution potential $W_{\alpha} * \mu$ can be explicitly computed.

What is even more surprising is that, for $0<\alpha<1$, the normalised characteristic function of $\Omega(\sqrt{1-\alpha}, \sqrt{1+\alpha})$ is actually the minimiser of the energy $I_{\alpha}$ and that it is possible to prove it. To see this, let us first consider the purely logarithmic case $\alpha=0$. By radial symmetry of the energy and uniqueness, the minimiser $\mu_{0}$ must be radial. This case is well-known to be connected to the classical obstacle problem for the Laplace operator $[10,12,13]$. Defining $\Psi_{0}=W_{0} * \mu_{0}$ and assuming that $\mu_{0}$ is supported on the closure of a smooth bounded open set $\Omega$, the Euler-Lagrange equations (1.5)-(1.6) imply

$$
\begin{cases}\Psi_{0} \geq C_{0}-\frac{|x|^{2}}{2} & \text { in } \mathbb{R}^{2} \\ -\Delta \Psi_{0} \geq 0 & \text { in } \mathbb{R}^{2} \\ \left(\Psi_{0}-C_{0}+\frac{|x|^{2}}{2}\right) \Delta \Psi_{0}=0 & \text { in } \mathbb{R}^{2}\end{cases}
$$

where $\bar{\Omega}$ is the coincidence set, i.e., the points where $\Psi_{0}=C_{0}-\left(|x|^{2} / 2\right)$. It is not surprising from (1.7) that $\mu_{0}$ is the normalised characteristic function of the coincidence set $\bar{\Omega}$ with constant density since $2 \pi \mu_{0}=-\Delta \Psi_{0}$, and due to the radial symmetry the Euclidean ball is the clear candidate for $\Omega$.

In the presence of the anisotropic term, that is, for $\alpha>0$, we write $W_{\alpha}=W_{0}+\alpha F$ and define $\Psi_{\alpha}=W_{\alpha} * \mu_{\alpha}$ where $\mu_{\alpha}$ is the unique minimiser of $I_{\alpha}$. A corresponding obstacle problem as (1.7) can be formally written for the potential $\Psi_{\alpha}$, and the coincidence set is again determined by the condition $\Psi_{\alpha}=C_{\alpha}-\left(|x|^{2} / 2\right)$. Assuming it is the closure of a smooth bounded open set $\Omega$, one obtains

$$
\Delta \Psi_{\alpha}=-2 \pi \mu_{\alpha}+\alpha \Delta F * \mu_{\alpha}=-2 \text { in } \Omega .
$$

If $\mu_{\alpha}$ is the normalised characteristic function of $\Omega$, then $\Delta F * \mu_{\alpha}$ should be constant on $\Omega$ as well. However, computing $\Delta F * \chi_{\Omega}$ for a general domain $\Omega$ is a highly non-trivial task, and in principle $\Delta F * \chi_{\Omega}$ could be a very complicated object. It is therefore surprising that, for elliptic domains $\Omega=\Omega(a, b), \Delta F * \chi_{\Omega(a, b)}$ is constant in $\Omega(a, b)$. In fact, as we mentioned before, we are able to compute the convolution potential $W_{\alpha} * \chi_{\Omega(a, b)}$ in the whole of $\mathbb{R}^{2}$, and to show that in $\Omega(a, b)$ it is a homogeneous polynomial of degree 2 plus a constant. From this property, indeed, establishing the first Euler-Lagrange condition is a relatively easy task. The expression of the convolution potential (and of its gradient) outside $\Omega(a, b)$ is instead much more involved, so that establishing the second Euler-Lagrange condition is the challenge.

1.2. Dimension of the support of the equilibrium measure. We have seen that the values $\alpha= \pm 1$ of the weight for the anisotropic term of the kernel $W_{\alpha}$ determine a sharp transition in the dimension of the support of the minimising measure $\mu_{\alpha}$ from two (for $\alpha \in(-1,1)$ ) to one (for $\alpha \leq-1$ and $\alpha \geq 1$ ). 
For general energies of the form

$$
E(\mu)=\frac{1}{2} \int_{\mathbb{R}^{d}} \int_{\mathbb{R}^{d}} W(x-y) d \mu(x) d \mu(y)+\int_{\mathbb{R}^{d}} V(x) d \mu(x) \text { for all } \mu \in \mathcal{P}\left(\mathbb{R}^{d}\right),
$$

where $W: \mathbb{R}^{d} \rightarrow \mathbb{R} \cup\{+\infty\}$ is an interaction potential and $V: \mathbb{R}^{d} \rightarrow \mathbb{R} \cup\{+\infty\}$ is a confining potential, understanding how the dimension of the support of the minimisers depends on $W$ and $V$ is a challenging question.

In [3] the authors showed that the dimension of the support of a minimiser of $E$ is directly related to the strength of the repulsion of the potential at the origin. What they showed is that the stronger the repulsion (up to Newtonian), the higher the dimension of the support. The case of mild repulsive potentials in which the minimisers are a finite number of Dirac deltas has been recently studied in [19].

Our result shows that a change of the dimension of the support of the minimisers can also be obtained by tuning the asymmetry of the interaction potential.

Another challenging question arising from the results in this paper and in [3] is to give explicit examples in which a change of the dimension of the support of the minimisers is obtained by tuning the confining potential $V$, or the singularity of the interaction potential at zero.

1.3. More general interactions and evolution. The problem of establishing existence and qualitative properties of minimisers of (1.8) has been the object of an intense research activity in the last 20 years; see, e.g., [16-18,22,41]. A very related question is to study the asymptotic stability properties of stationary solutions of its associated gradient flow

$$
\partial_{t} \mu=\operatorname{div}\left(\mu \nabla \frac{\delta E}{\delta \mu}\right)=\operatorname{div}(\mu \nabla(W * \mu+V)) \text { on } \mathbb{R}^{d}, \text { for } t>0,
$$

in the Wasserstein sense $[2,21]$, where $\mu:[0, \infty) \rightarrow \mathcal{P}\left(\mathbb{R}^{d}\right)$ is a curve in the space of probability measures. Here, the variational derivative $\delta E / \delta \mu:=W * \mu+V$ is obtained by doing variations of the energy $E(\mu)$ preserving the unit mass of the measure as originally introduced in [38]; see $[2,45]$ for the general theory. Equations like (1.9) describe the macroscopic behaviour of agents interacting via a potential $W$, and are at the core of many applications ranging from mathematical biology to economics; see [7,29,35,43] and the references therein.

In most of the early works, interaction and confinement potentials were assumed to be smooth enough and convex in some sense, including interesting cases with applications in granular media modelling $[21,44]$. In most of the applications however the potential $W$ is singular, and in fact most of the rich structure of the minimisers happens when the potentials are singular at the origin; see [1,3,5,6,20,24,31,40] and [22] for a recent review in the subject. Typical interaction potentials in applications are repulsive at the origin and attractive at infinity (the latter guaranteeing confinement). The classical case corresponding to $\alpha=0$ in (1.2), that is, the repulsive Newtonian interaction with quadratic confinement, was analysed in [6]. They showed that all the solutions of the corresponding gradient flow equation (1.9) converge as $t \rightarrow \infty$ to the suitably normalised characteristic function of an Euclidean ball with a certain radius. In particular, their results imply a dynamic proof of the classical minimisation result of Frostman [26,39]. We also mention [40], where these results are recovered by taking the limit from the fractional diffusion range and the obstacle problems studied in $[14,15]$. 
Euler-Lagrange necessary conditions for local minimisers of the energy $E$ in a suitable topology were derived in [3], see also [39] for the particular case of the logarithmic potential. They were used to give necessary and sufficient conditions on repulsiveattractive potentials to have existence of global minimisers [16,41], and to analyse their regularity for potentials that are as repulsive as, or more singular than, the Newtonian potential [18]. In both cases, global minimisers are solutions of some related obstacle problems for Laplacian or nonlocal fractional Laplacian operators, implying that they are bounded and smooth in their support, or even continuous up to the boundary $[10,13,18,22]$. Similar Euler-Lagrange conditions were also used for nonlinear versions of the Keller-Segel model in order to characterise minimisers of related functionals [17].

The plan of the paper is as follows. The proof of the Euler-Lagrange conditions in Theorem 1.1 will be done in Sect. 3. We start next section, Sect. 2, by showing the existence and uniqueness of the global minimiser for $I_{\alpha}$. Section 4 contains some additional information. On the one hand, we discuss an alternative proof of the first Euler-Lagrange condition and compute the minimal energy. On the other hand, we study more general anisotropies.

\section{Existence and Uniqueness of the Minimiser of $I_{\alpha}$}

In this section we prove that for every $\alpha \in \mathbb{R}$ the nonlocal energy $I_{\alpha}$ defined in (1.1) has a unique minimiser $\mu_{\alpha} \in \mathcal{P}\left(\mathbb{R}^{2}\right)$, and that the minimiser has a compact support.

We observe that it is sufficient to consider the case $\alpha \in(0,1)$. In fact, for $\alpha=0$, that is, for purely logarithmic interactions, it is well-known that there exists a unique minimiser of $I_{0}$, which is given by the so-called circle law $\mu_{0}:=\frac{1}{\pi} \chi_{B_{1}(0)}$ (see, e.g., $[26,39]$, and the references therein). The case $\alpha=1$, that is, the case of interacting edge dislocations, has been recently solved in [37], and it has been shown that $I_{1}$ has a unique minimiser, given by the semi-circle law (1.3). A simple comparison argument shows that $\mu_{1}$ is indeed the unique minimiser of $I_{\alpha}$ for any $\alpha \geq 1$. In fact, for any $\alpha \geq 1$ and any $\mu \in \mathcal{P}\left(\mathbb{R}^{2}\right)$ with $\mu \neq \mu_{1}$, we have

$$
I_{\alpha}\left(\mu_{1}\right)=I_{1}\left(\mu_{1}\right)<I_{1}(\mu) \leq I_{\alpha}(\mu)
$$

If $\alpha<0$, instead, we observe that

$$
W_{\alpha}\left(x_{1}, x_{2}\right)=-\log |x|+|\alpha| \frac{x_{2}^{2}}{|x|^{2}}+\alpha,
$$

hence all results in this case may be obtained from those with $\alpha>0$ just by swapping $x_{1}$ and $x_{2}$.

In what follows we assume the kernel $W_{\alpha}$ to be extended to the whole of $\mathbb{R}^{2}$ by continuity, that is, we set $W_{\alpha}(0):=+\infty$.

Proposition 2.1. Let $\alpha \in[0,1]$. Then the energy $I_{\alpha}$ is well defined on $\mathcal{P}\left(\mathbb{R}^{2}\right)$, is strictly convex on the class of measures with compact support and finite interaction energy, and has a unique minimiser in $\mathcal{P}\left(\mathbb{R}^{2}\right)$. Moreover, the minimiser has compact support and finite energy.

Proof. The case $\alpha=0$ is well-known. The proof for $\alpha \in(0,1)$ follows the lines of the analogous result for $\alpha=1$; see [37, Section 2]. For the convenience of the reader we recall the main steps of the proof. 
Step 1: Existence of a compactly supported minimiser. We have that

$$
W_{\alpha}(x-y)+\frac{1}{2}\left(|x|^{2}+|y|^{2}\right) \geq W_{0}(x-y)+\frac{1}{2}\left(|x|^{2}+|y|^{2}\right) \geq\left(\frac{1}{2}-\frac{1}{e}\right)\left(|x|^{2}+|y|^{2}\right) .
$$

The lower bound (2.1) guarantees that $I_{\alpha}$ is well defined and nonnegative on $\mathcal{P}\left(\mathbb{R}^{2}\right)$ and, since $I_{\alpha}\left(\mu_{0}\right)<+\infty$, where $\mu_{0}=\frac{1}{\pi} \chi_{B_{1}(0)}$, it implies that $\inf _{\mathcal{P}\left(\mathbb{R}^{2}\right)} I_{\alpha}<+\infty$. It also provides tightness and hence compactness with respect to narrow convergence for minimising sequences, that, together with the lower semicontinuity of $I_{\alpha}$, guarantees the existence of a minimiser.

As in [37, Section 2.2], one can show that any minimiser of $I_{\alpha}$ has compact support, again by (2.1).

Step 2: Strict convexity of $I_{\alpha}$ and uniqueness of the minimiser. We prove that

$$
\int_{\mathbb{R}^{2}} W_{\alpha} *\left(v_{1}-v_{2}\right) d\left(v_{1}-v_{2}\right)>0
$$

for every $v_{1}, v_{2} \in \mathcal{P}\left(\mathbb{R}^{2}\right), v_{1} \neq v_{2}$, with compact support and finite interaction energy, namely such that $\int_{\mathbb{R}^{2}}\left(W_{\alpha} * v_{i}\right) d v_{i}<+\infty$ for $i=1$, 2. Condition (2.2) implies strict convexity of $I_{\alpha}$ on the set of probability measures with compact support and finite interaction energy and, consequently, uniqueness of the minimiser.

To prove (2.2), we argue again as in [37, Section 2.3]. The heuristic idea is to rewrite the interaction energy of $v:=v_{1}-v_{2}$ in Fourier space, as

$$
\int_{\mathbb{R}^{2}} W_{\alpha} * v d v=\int_{\mathbb{R}^{2}} \hat{W}_{\alpha}(\xi)|\hat{v}(\xi)|^{2} d \xi
$$

Since $v$ is a neutral measure, $\hat{v}$ vanishes at $\xi=0$. So, the claim (2.2) follows by showing the positivity of the Fourier transform of $W_{\alpha}$ on positive test functions vanishing at zero.

Since $W_{\alpha} \in L_{\text {loc }}^{1}\left(\mathbb{R}^{2}\right)$ and has a logarithmic growth at infinity, it is a tempered distribution, namely $W_{\alpha} \in \mathcal{S}^{\prime}$, where $\mathcal{S}$ denotes the Schwartz space; hence $\hat{W}_{\alpha} \in \mathcal{S}^{\prime}$. We recall that $\hat{W}_{\alpha}$ is defined by the formula

$$
\left\langle\hat{W}_{\alpha}, \varphi\right\rangle:=\left\langle W_{\alpha}, \hat{\varphi}\right\rangle \quad \text { for every } \varphi \in \mathcal{S}
$$

where, for $\xi \in \mathbb{R}^{2}$,

$$
\hat{\varphi}(\xi):=\int_{\mathbb{R}^{2}} \varphi(x) e^{-2 \pi i \xi \cdot x} d x .
$$

Proceeding as in [37, Section 2.3], we have that the Fourier transform $\hat{W}_{\alpha}$ of $W_{\alpha}$ is given by

$$
\begin{aligned}
\left\langle\hat{W}_{\alpha}, \varphi\right\rangle=( & \left.\frac{\alpha}{2}+\gamma+\log \pi\right) \varphi(0)+\frac{1}{2 \pi} \int_{|\xi| \leq 1}(\varphi(\xi)-\varphi(0)) \frac{(1-\alpha) \xi_{1}^{2}+(1+\alpha) \xi_{2}^{2}}{|\xi|^{4}} d \xi \\
& +\frac{1}{2 \pi} \int_{|\xi|>1} \varphi(\xi) \frac{(1-\alpha) \xi_{1}^{2}+(1+\alpha) \xi_{2}^{2}}{|\xi|^{4}} d \xi
\end{aligned}
$$

for every $\varphi \in \mathcal{S}$, where $\gamma$ is the Euler constant. In particular, from (2.3), we have that

$$
\left\langle\hat{W}_{\alpha}, \varphi\right\rangle=\frac{1}{2 \pi} \int_{\mathbb{R}^{2}} \frac{(1-\alpha) \xi_{1}^{2}+(1+\alpha) \xi_{2}^{2}}{|\xi|^{4}} \varphi(\xi) d \xi
$$


for every $\varphi \in \mathcal{S}$ with $\varphi(0)=0$. Thus, (2.4) implies that $\left\langle\hat{W}_{\alpha}, \varphi\right\rangle>0$ for every $\varphi \in \mathcal{S}$ with $\varphi(0)=0$ and $\varphi \geq 0, \varphi \not \equiv 0$.

Finally, the approximation argument in the proof of [37, Theorem 1.1] allows one to pass from test functions in $\mathcal{S}$ to measures. Hence (2.2) is proved.

\section{Characterisation of the Minimiser of $I_{\alpha}$ : The Ellipse Law.}

It is a standard computation in potential theory (see [37,39]) to show that any minimiser $\mu$ of $I_{\alpha}$ must satisfy the following Euler-Lagrange conditions: there exists $C \in \mathbb{R}$ such that

$$
\begin{aligned}
& \left(W_{\alpha} * \mu\right)(x)+\frac{|x|^{2}}{2}=C \text { for } \mu \text {-a.e. } x \in \operatorname{supp} \mu, \\
& \left(W_{\alpha} * \mu\right)(x)+\frac{|x|^{2}}{2} \geq C \text { for q.e. } x \in \mathbb{R}^{2},
\end{aligned}
$$

where quasi everywhere (q.e.) means up to sets of zero capacity. The Euler-Lagrange conditions (3.1)-(3.2) are in fact equivalent to minimality for $0 \leq \alpha \leq 1$ due to Proposition 2.1. See [37, Section 3] for details.

In this section we show that, for every $0 \leq \alpha<1$, the measure $\mu_{\alpha}$ defined in (1.4) satisfies the Euler-Lagrange conditions (1.5)-(1.6), for some constant $C_{\alpha} \in \mathbb{R}$. By the above discussion this immediately implies that $\mu_{\alpha}$ is the unique minimiser of $I_{\alpha}$, thus completing the proof of Theorem 1.1. The precise value of $C_{\alpha}$ will be computed in Sect. 4.

We begin by studying $W_{\alpha} * \chi_{\Omega(a, b)}$ for every $b \geq a>0$. We note that the function $W_{\alpha} * \chi_{\Omega(a, b)}$ is $C^{1}$ in $\mathbb{R}^{2}$ (see [42]). As a first step, we compute the convolution $\left(\nabla W_{\alpha} *\right.$ $\left.\chi_{\Omega(a, b)}\right)(x)$ for every $b \geq a>0$ and at every point $x \in \mathbb{R}^{2}$.

In fact we wish to prove that $\mu_{\alpha}$ satisfies the conditions

$$
\begin{aligned}
\nabla\left(W_{\alpha} * \mu_{\alpha}\right)(x)+x=0 & \text { for every } x \in \Omega(\sqrt{1-\alpha}, \sqrt{1+\alpha}), \\
x \cdot \nabla\left(W_{\alpha} * \mu_{\alpha}\right)(x)+|x|^{2} \geq 0 & \text { for every } x \in \mathbb{R}^{2} .
\end{aligned}
$$

Clearly, conditions (3.3)-(3.4) imply that (1.5)-(1.6) are satisfied for some constant $C_{\alpha} \in \mathbb{R}$.

In order to evaluate the convolution $\nabla W_{\alpha} * \chi_{\Omega(a, b)}$, it is convenient to work in complex variables. As usual, we identify $z=x_{1}+i x_{2} \equiv x=\left(x_{1}, x_{2}\right)$, and we write the standard differential operators as

$$
\partial=\frac{\partial}{\partial z}=\frac{1}{2}\left(\frac{\partial}{\partial x_{1}}-i \frac{\partial}{\partial x_{2}}\right) \text { and } \bar{\partial}=\frac{\partial}{\partial \bar{z}}=\frac{1}{2}\left(\frac{\partial}{\partial x_{1}}+i \frac{\partial}{\partial x_{2}}\right) .
$$

In complex variables the potential $W_{\alpha}$ in (1.2) reads as

$$
W_{\alpha}(x) \equiv W_{\alpha}(z)=-\frac{1}{2} \log (z \bar{z})+\frac{\alpha}{2}\left(1+\frac{z}{2 \bar{z}}+\frac{\bar{z}}{2 z}\right),
$$

and thus

$$
\nabla W_{\alpha}(x)=-\frac{x}{|x|^{2}}+2 \alpha \frac{x_{1} x_{2}}{|x|^{4}} x^{\perp} \equiv 2 \bar{\partial} W_{\alpha}(z)=-\frac{1}{\bar{z}}+\frac{\alpha}{2} \frac{1}{z}-\frac{\alpha}{2} \frac{z}{\bar{z}^{2}},
$$

where $x^{\perp}=\left(x_{2},-x_{1}\right)$.

The result is the following. 
Proposition 3.1. Let $b \geq a>0$ and $\mu_{a, b}:=\frac{1}{\pi a b} \chi_{\Omega(a, b)}$ be the (normalised) characteristic function of the ellipse of semi-axes $a$ and $b$. Then we have

$$
\begin{aligned}
\nabla\left(W_{\alpha} * \mu_{a, b}\right)(z) & =\frac{1}{\pi a b}\left(-\frac{1}{\bar{z}}+\frac{\alpha}{2} \frac{1}{z}-\frac{\alpha}{2} \frac{z}{\bar{z}^{2}}\right) * \chi_{\Omega(a, b)}(z) \\
& =\frac{1}{a b}(-1-\alpha \lambda) z+\frac{1}{a b}\left(\lambda+\frac{\alpha}{2}+\lambda^{2} \frac{\alpha}{2}\right) \bar{z}
\end{aligned}
$$

for every $z \in \Omega(a, b)$ and

$$
\begin{aligned}
\nabla\left(W_{\alpha} * \mu_{a, b}\right)(z) & =\frac{1}{\pi a b}\left(-\frac{1}{\bar{z}}+\frac{\alpha}{2} \frac{1}{z}-\frac{\alpha}{2} \frac{z}{\bar{z}^{2}}\right) * \chi_{\Omega(a, b)}(z) \\
& =-(2+\alpha \lambda) h(\bar{z})+\alpha h(z)-\alpha(\lambda \bar{z}-z+2 a b h(\bar{z})) h^{\prime}(\bar{z})
\end{aligned}
$$

for every $z \in \Omega(a, b)^{c}$. Here

$$
\lambda:=\frac{a-b}{a+b}, \quad h(z):=\frac{1}{z+\sqrt{z^{2}+c^{2}}},
$$

and $c^{2}=b^{2}-a^{2}$, where $c$ is the eccentricity of the ellipse.

We note that here and in what follows $\sqrt{z^{2}+c^{2}}$ denotes the branch of the complex square root that behaves asymptotically as $z$ at infinity. Namely, for $z \in \mathbb{C} \backslash[-i c, i c]$ such that $z=\rho e^{i \theta}$ with $\rho>0$ and $0 \leq \theta<2 \pi$, we have $z^{2}+c^{2}=\rho_{1} e^{i \theta_{1}}$ with $\rho_{1}>0$, and $0 \leq \theta_{1}<2 \pi$ if $0 \leq \theta<\pi$ and $2 \pi \leq \theta_{1}<4 \pi$ if $\pi \leq \theta<2 \pi$, and $\sqrt{z^{2}+c^{2}}=\sqrt{\rho_{1}} e^{i \theta_{1} / 2}$. In other words, we choose the branch of the complex square root that preserves the quadrants. In particular, for every $z \in \mathbb{C} \backslash[-i c, i c]$ we have $\Re(z) \Re\left(\sqrt{z^{2}+c^{2}}\right) \geq 0$ and $\Im(z) \Im\left(\sqrt{z^{2}+c^{2}}\right) \geq 0$, where $\Re(z)$ and $\Im(z)$ denote, respectively, the real and imaginary part of $z$. This property will be crucial in the proof of Theorem 1.1.

Proof of Proposition 3.1. We divide the proof into two steps.

Step 1: Computation of $\frac{1}{z} * \chi_{\Omega(a, b)}$ and $\frac{1}{\bar{z}} * \chi_{\Omega(a, b)}$. We observe that $\frac{1}{z} * \chi_{\Omega(a, b)}$ is the Cauchy transform of the (characteristic function of the) ellipse $\Omega(a, b)$, up to a multiplicative constant. Indeed, the Cauchy transform of a $C^{1}$ domain $\Omega \subset \mathbb{C}$ is defined as

$$
\mathcal{C}\left(\chi_{\Omega}\right)(z):=\frac{1}{\pi} \int_{\Omega} \frac{1}{z-\xi} d \xi .
$$

Clearly $\mathcal{C}\left(\chi_{\Omega}\right)$ is a continuous function in $\mathbb{C}$, holomorphic in $\mathbb{C} \backslash \bar{\Omega}$ and vanishes at infinity.

In the special case of an ellipse, namely for $\Omega=\Omega(a, b)$, the expression (3.9) can be computed explicitly (see [28, page 1408]), and is given by

$$
\frac{1}{\pi z} * \chi_{\Omega(a, b)}=\mathcal{C}\left(\chi_{\Omega(a, b)}\right)(z)= \begin{cases}\bar{z}-\lambda z & \text { if } z \in \Omega(a, b), \\ 2 a b h(z) & \text { if } z \in \Omega(a, b)^{c},\end{cases}
$$

where $\lambda$ and $h$ are as in (3.8).

By taking the conjugate of (3.10) we obtain directly

$$
\frac{1}{\pi \bar{z}} * \chi_{\Omega(a, b)}=\mathcal{C}\left(\chi_{\Omega(a, b)}\right)(\bar{z})= \begin{cases}z-\lambda \bar{z} & \text { if } z \in \Omega(a, b), \\ 2 a b h(\bar{z}) & \text { if } z \in \Omega(a, b)^{c},\end{cases}
$$


and hence the first two terms of $\nabla W_{\alpha} * \chi_{\Omega(a, b)}$ are now computed. Step 2: Computation of $\frac{z}{\bar{z}^{2}} * \chi_{\Omega(a, b)}$. We start by observing that

$$
-\frac{1}{\pi} \frac{z}{\bar{z}^{2}} * \chi_{\Omega(a, b)}=\bar{\partial}\left(\frac{1}{\pi} \frac{z}{\bar{z}} * \chi_{\Omega(a, b)}\right),
$$

hence it is sufficient to compute $\frac{1}{\pi} \frac{z}{\bar{z}} * \chi_{\Omega(a, b)}$. Now we recall that $\frac{1}{\pi} \frac{z}{\bar{z}}$ is the fundamental solution of $\partial^{2}$, hence

$$
\partial^{2}\left(\frac{1}{\pi} \frac{z}{\bar{z}} * \chi_{\Omega(a, b)}\right)=\partial^{2}\left(\frac{1}{\pi} \frac{z}{\bar{z}}\right) * \chi_{\Omega(a, b)}= \begin{cases}1 & \text { if } z \in \Omega(a, b), \\ 0 & \text { if } z \in \Omega(a, b)^{c} .\end{cases}
$$

The previous expression implies that $\frac{1}{\pi} \frac{z}{\bar{z}} * \chi_{\Omega(a, b)}$ is quadratic in $z$ in $\Omega(a, b)$ and linear in $z$ in $\Omega(a, b)^{c}$. More precisely,

$$
\frac{1}{\pi} \frac{z}{\bar{z}} * \chi_{\Omega(a, b)}= \begin{cases}\frac{z^{2}}{2}+z h_{1}^{i}(\bar{z})+h_{2}^{i}(\bar{z}) & \text { if } z \in \Omega(a, b) \\ z h_{1}^{o}(\bar{z})+h_{2}^{o}(\bar{z}) & \text { if } z \in \Omega(a, b)^{c}\end{cases}
$$

with $h_{1}^{i}, h_{2}^{i}, h_{1}^{o}$ and $h_{2}^{o}$ holomorphic functions in their respective domains (and the indices $i$ and $o$ stand for "inner" and "outer").

It remains to determine the functions $h_{1}^{i}, h_{2}^{i}, h_{1}^{o}$ and $h_{2}^{o}$ explicitly. By applying the operator $\partial$ to both sides of (3.13), we deduce

$$
\frac{1}{\pi \bar{z}} * \chi_{\Omega(a, b)}= \begin{cases}z+h_{1}^{i}(\bar{z}) & \text { if } z \in \Omega(a, b), \\ h_{1}^{o}(\bar{z}) & \text { if } z \in \Omega(a, b)^{c},\end{cases}
$$

which, together with (3.11), leads to the identification of $h_{1}^{i}$ and $h_{1}^{o}$, as

$$
h_{1}^{i}(\bar{z})=-\lambda \bar{z} \text { in } \Omega(a, b) \text { and } h_{1}^{o}(\bar{z})=2 a b h(\bar{z}) \text { in } \Omega(a, b)^{c} .
$$

Substituting these expressions into (3.13) we then have

$$
\frac{1}{\pi} \frac{z}{\bar{z}} * \chi_{\Omega(a, b)}= \begin{cases}\frac{z^{2}}{2}-\lambda \bar{z} z+h_{2}^{i}(\bar{z}) & \text { if } z \in \Omega(a, b) \\ 2 a b z h(\bar{z})+h_{2}^{o}(\bar{z}) & \text { if } z \in \Omega(a, b)^{c}\end{cases}
$$

with $h_{2}^{i}$ and $h_{2}^{o}$ holomorphic functions in their respective domains, still to be determined. By (3.12), however, it is sufficient to determine their derivatives, since, by applying the operator $\bar{\partial}$ to both sides of (3.14), we have

$$
-\frac{1}{\pi} \frac{z}{\bar{z}^{2}} * \chi_{\Omega(a, b)}=\bar{\partial}\left(\frac{1}{\pi} \frac{z}{\bar{z}}\right) * \chi_{\Omega(a, b)}= \begin{cases}-\lambda z+\left(h_{2}^{i}\right)^{\prime}(\bar{z}) & \text { if } z \in \Omega(a, b), \\ 2 a b z h^{\prime}(\bar{z})+\left(h_{2}^{o}\right)^{\prime}(\bar{z}) & \text { if } z \in \Omega(a, b)^{c} .\end{cases}
$$

Now we observe that the function $\frac{1}{\pi} \frac{z}{\bar{z}^{2}} * \chi_{\Omega(a, b)}$ on the left-hand side of (3.15) is continuous in $\mathbb{C}$ and decays to zero as $z \rightarrow \infty$; see, e.g., [42]. Therefore, also the right-hand side of (3.15) is continuous in $\mathbb{C}$, which implies in particular that

$$
-\lambda z+\left(h_{2}^{i}\right)^{\prime}(\bar{z})=2 a b z h^{\prime}(\bar{z})+\left(h_{2}^{o}\right)^{\prime}(\bar{z})
$$


for every $z \in \partial \Omega(a, b)$. By using the expression of the boundary of the ellipse in complex variables, namely

$$
\partial \Omega(a, b)=\{z \in \mathbb{C}: \bar{z}=\lambda z+2 a b h(z)\}
$$

where $\lambda$ and $h$ are defined as in (3.8), and by rearranging the terms in (3.16), we obtain that

$$
-\lambda^{2} \bar{z}+\left(h_{2}^{i}\right)^{\prime}(\bar{z})=2 a b \lambda h(\bar{z})+2 a b(\lambda \bar{z}+2 a b h(\bar{z})) h^{\prime}(\bar{z})+\left(h_{2}^{o}\right)^{\prime}(\bar{z})
$$

on $\partial \Omega(a, b)$. Consider now the auxiliary function

$$
R(\bar{z})= \begin{cases}-\lambda^{2} \bar{z}+\left(h_{2}^{i}\right)^{\prime}(\bar{z}) & \text { if } z \in \Omega(a, b), \\ 2 a b \lambda h(\bar{z})+2 a b(\lambda \bar{z}+2 a b h(\bar{z})) h^{\prime}(\bar{z})+\left(h_{2}^{o}\right)^{\prime}(\bar{z}) & \text { if } z \in \Omega(a, b)^{c} .\end{cases}
$$

Because of the continuity condition (3.17), $R(\bar{z})$ is an anti-holomorphic function in $\mathbb{C}$. Moreover, it easy to see that $R$ has zero limit at $\infty$. This is clear for all the terms in the expression of $R$ in $\Omega(a, b)^{c}$ involving $h$ and $h^{\prime}$, by (3.8); for the term $\left(h_{2}^{o}\right)^{\prime}$ it follows by (3.15). The Liouville Theorem then implies that $R(\bar{z}) \equiv 0$. As a consequence, both expressions on the right-hand side of (3.18) are zero, which gives

$$
\begin{aligned}
& \left(h_{2}^{i}\right)^{\prime}(\bar{z})=\lambda^{2} \bar{z} \text { in } \Omega(a, b) \text { and } \\
& \left(h_{2}^{o}\right)^{\prime}(\bar{z})=-2 a b \lambda h(\bar{z})-2 a b(\lambda \bar{z}+2 a b h(\bar{z})) h^{\prime}(\bar{z}) \text { in } \Omega(a, b)^{c},
\end{aligned}
$$

and hence the identification of $\left(h_{2}^{i}\right)^{\prime}$ and $\left(h_{2}^{o}\right)^{\prime}$ in their respective domains.

Plugging these formulas into (3.15), we finally conclude that

$$
-\frac{1}{\pi} \frac{z}{\bar{z}^{2}} * \chi_{\Omega(a, b)}= \begin{cases}\lambda^{2} \bar{z}-\lambda z & \text { if } z \in \Omega(a, b), \\ 2 a b(z-\lambda \bar{z}-2 a b h(\bar{z})) h^{\prime}(\bar{z})-2 a b \lambda h(\bar{z}) & \text { if } z \in \Omega(a, b)^{c} .\end{cases}
$$

Finally, using (3.10), (3.11) and (3.19) we have that (3.6) and (3.7) immediately follow.

We are now in the position to prove our main result.

Proof of Theorem 1.1. We need to show that (3.3)-(3.4) hold. By using the expression (3.5), conditions (3.3)-(3.4) are equivalent to show that for $a=\sqrt{1-\alpha}$ and $b=\sqrt{1+\alpha}$ we have

$$
\begin{aligned}
\frac{1}{\pi a b}\left(\nabla W_{\alpha} * \chi_{\Omega(a, b)}\right)(z)+z & =0 \quad \text { for every } z \in \Omega(a, b), \\
\frac{1}{\pi a b} \Re\left(\bar{z}\left(\nabla W_{\alpha} * \chi_{\Omega(a, b)}\right)(z)\right)+|z|^{2} \geq 0 & \text { for every } z \in \Omega(a, b)^{c} .
\end{aligned}
$$

Step 1: The measure $\mu_{\alpha}$ satisfies (3.3). Using (3.6), we have that

$$
\frac{1}{\pi a b}\left(\nabla W_{\alpha} * \chi_{\Omega(a, b)}\right)(z)+z=\frac{1}{a b}(-1-\alpha \lambda+a b) z+\frac{1}{a b}\left(\lambda+\frac{\alpha}{2}+\lambda^{2} \frac{\alpha}{2}\right) \bar{z}
$$

for every $z \in \Omega(a, b)$. It is easy to check that $a=\sqrt{1-\alpha}$ and $b=\sqrt{1+\alpha}$ are the unique solution of the system

$$
\left\{\begin{array}{l}
-1-\alpha \lambda+a b=0 \\
\lambda+\frac{\alpha}{2}+\lambda^{2} \frac{\alpha}{2}=0
\end{array}\right.
$$


leading to condition (3.20), hence (3.3).

Step 2: The measure $\mu_{\alpha}$ satisfies (3.4). By (3.7) we have that

$$
\left(\nabla W_{\alpha} * \mu_{\alpha}\right)(z)+z=-(2+\alpha \lambda) h(\bar{z})+\alpha h(z)-\alpha(\lambda \bar{z}-z+2 a b h(\bar{z})) h^{\prime}(\bar{z})+z
$$

for every $z \in \Omega(\sqrt{1-\alpha}, \sqrt{1+\alpha})^{c}$. Since $a=\sqrt{1-\alpha}$ and $b=\sqrt{1+\alpha}$, we note that

$$
\lambda=\frac{1}{\alpha}\left(\sqrt{1-\alpha^{2}}-1\right) \quad \text { and } \quad a b=\sqrt{1-\alpha^{2}} .
$$

To simplify the expression (3.22) we also observe that

$$
h(z)=\frac{1}{z+\sqrt{z^{2}+2 \alpha}}=\frac{1}{2 \alpha}\left(\sqrt{z^{2}+2 \alpha}-z\right) \quad \text { and } \quad h^{\prime}(z)=\frac{-h(z)}{\sqrt{z^{2}+2 \alpha}},
$$

where we have used the fact that $c^{2}=b^{2}-a^{2}=2 \alpha$. Substituting (3.23) and (3.24) into (3.22), and performing some simple algebraic manipulations, we deduce that

$$
\begin{aligned}
\left(\nabla W_{\alpha} * \mu_{\alpha}\right)(z)+z & =\frac{1}{2} \sqrt{z^{2}+2 \alpha}-\frac{1}{2 \alpha} \sqrt{\bar{z}^{2}+2 \alpha}+\frac{\bar{z}}{2}\left(z+\frac{1}{\alpha} \bar{z}\right) \frac{1}{\sqrt{\bar{z}^{2}+2 \alpha}} \\
& =\frac{\left|z^{2}+2 \alpha\right|+\left|z^{2}\right|-2}{2\left|z^{2}+2 \alpha\right|} \sqrt{z^{2}+2 \alpha}
\end{aligned}
$$

Proving that (3.21) holds with $a=\sqrt{1-\alpha}$ and $b=\sqrt{1+\alpha}$ is then equivalent to showing that

$$
\frac{\left|z^{2}+2 \alpha\right|+\left|z^{2}\right|-2}{2\left|z^{2}+2 \alpha\right|} \Re\left(\bar{z} \sqrt{z^{2}+2 \alpha}\right) \geq 0 \text { for every } z \in \Omega(\sqrt{1-\alpha}, \sqrt{1+\alpha})^{c} .
$$

Now, we recall that $\sqrt{z^{2}+2 \alpha}$ denotes the branch of the complex square root preserving the quadrants, that is, for every $z \in \mathbb{C} \backslash[-i \sqrt{2 \alpha}, i \sqrt{2 \alpha}]$ we have $\Re(z) \Re\left(\sqrt{z^{2}+2 \alpha}\right) \geq 0$ and $\Im(z) \Im\left(\sqrt{z^{2}+2 \alpha}\right) \geq 0$. Therefore, we immediately deduce that $\Re\left(\bar{z} \sqrt{z^{2}+2 \alpha}\right) \geq 0$ for every $z \in \Omega(\sqrt{1-\alpha}, \sqrt{1+\alpha})^{c}$.

To conclude the proof of the claim (3.25) it remains to show that $\left|z^{2}+2 \alpha\right|+\left|z^{2}\right|-2 \geq 0$ in $\Omega(\sqrt{1-\alpha}, \sqrt{1+\alpha})^{c}$. This is true since $\left|z^{2}+2 \alpha\right|+|z|^{2}-2$ is a level-set function for the ellipse $\Omega(\sqrt{1-\alpha}, \sqrt{1+\alpha})$. This is a general statement for ellipses $\Omega(a, b)$ with $b \geq a>0$, that we prove in Lemma 3.2 below.

The proof of Theorem 1.1 is thus complete, up to the computation of the constant $C_{\alpha}$, that we postpone to Sect. 4 .

Lemma 3.2. Let $\Gamma=\partial \Omega(a, b)$, with $b \geq a>0$. Then

$$
\begin{aligned}
& \left|z^{2}\right|+\left|z^{2}+c^{2}\right|=a^{2}+b^{2} \quad \text { if } z \in \Gamma, \\
& \left|z^{2}\right|+\left|z^{2}+c^{2}\right| \geq a^{2}+b^{2} \quad \text { if } z \in \Omega(a, b)^{c},
\end{aligned}
$$

where $c^{2}=b^{2}-a^{2}$. 
Proof. By dilating $z$ by a factor of $\frac{2}{a+b}$ we can further assume that $a+b=2$, and write $a=1-\beta$ and $b=1+\beta$, for some $0 \leq \beta<1$. Thus $c^{2}=(1+\beta)^{2}-(1-\beta)^{2}=4 \beta$ and $a^{2}+b^{2}=(1-\beta)^{2}+(1+\beta)^{2}=2\left(1+\beta^{2}\right)$, and the claim becomes

$$
\begin{array}{ll}
\left|z^{2}\right|+\left|z^{2}+4 \beta\right|=2\left(1+\beta^{2}\right) & \text { if } z \in \Gamma, \\
\left|z^{2}\right|+\left|z^{2}+4 \beta\right| \geq 2\left(1+\beta^{2}\right) & \text { if } z \in \Omega(1-\beta, 1+\beta)^{c} .
\end{array}
$$

Since $\Gamma=\{z=\zeta-\beta \bar{\zeta}:|\zeta|=1\}$, we have

$$
z^{2}=\left(\zeta^{2}+\beta^{2} \bar{\zeta}^{2}\right)-2 \beta
$$

and

$$
z^{2}+4 \beta=\left(\zeta^{2}+\beta^{2} \bar{\zeta}^{2}\right)+2 \beta .
$$

Now we observe that, whenever $\zeta \in \mathbb{C},|\zeta|=1$, then $\zeta^{2}+\beta^{2} \bar{\zeta}^{2} \in \partial \Omega\left(1+\beta^{2}, 1-\beta^{2}\right)$. Hence, since the foci of the ellipse $\partial \Omega\left(1+\beta^{2}, 1-\beta^{2}\right)$ are $\pm 2 \beta$, we deduce by (3.30)(3.31) that

$$
|z|^{2}+\left|z^{2}+4 \beta\right|=2\left(1+\beta^{2}\right),
$$

which completes the proof of (3.28) (and then of (3.26)). The statement (3.27) can be proved in the same way.

The limiting case $\alpha=1$ studied in [37] can be obtained from our analysis, valid for $0 \leq \alpha<1$, by means of a simple argument based on $\Gamma$-convergence. As a first step, we note that $\left(I_{\alpha}\right)_{\alpha \in(0,1)}$ is an increasing family of lower semicontinuous functionals (with respect to the narrow convergence of measures). Hence, $I_{1}$ is not only the pointwise limit of $I_{\alpha}$ as $\alpha \rightarrow 1^{-}$, but also the $\Gamma$-limit, namely

$$
\Gamma-\lim _{\alpha \rightarrow 1^{-}} I_{\alpha}=I_{1}
$$

see, e.g., [23, Proposition 5.4]. Let now $\mu_{\alpha}$ and $\mu_{1}$ be the measures defined in (1.4) and in (1.3), respectively. Since $\mu_{\alpha}$ is a minimiser of $I_{\alpha}$ for every $\alpha \in(0,1)$, and since $\mu_{\alpha} \rightarrow \mu_{1}$ narrowly as $\alpha \rightarrow 1^{-}$, the Fundamental Theorem of $\Gamma$-convergence implies that $\mu_{1}$ is a minimiser of $I_{1}$. It is in fact the unique minimiser, by the strict convexity of $I_{1}$.

Corollary 3.3. The unique minimiser of $I_{1}$ is given by the semi-circle law

$$
\mu_{1}:=\frac{1}{\pi} \delta_{0} \otimes \sqrt{2-x_{2}^{2}} \mathcal{H}^{1}\llcorner(-\sqrt{2}, \sqrt{2}) .
$$

\section{Further Comments}

4.1. Stationarity of $\mu_{\alpha}$ : an alternative proof. Here, we provide an alternative proof of the fact that, for every $\alpha \in(0,1)$, the ellipse-law $\mu_{\alpha}$ in (1.4) is a stationary solution of the gradient flow (1.9) associated to (1.5), namely it satisfies the Euler-Lagrange condition (1.5) inside its support, for some constant $C_{\alpha} \in \mathbb{R}$. In doing so, we also compute the exact value of $C_{\alpha}$ and the minimum value of $I_{\alpha}$. Finally, we explicitly compute the function $W_{\alpha} * \mu_{a, b}$ in the whole of $\mathbb{R}^{2}$ for a general ellipse (see Remark 4.1). We recall that $\mu_{a, b}=\frac{1}{\pi a b} \chi_{\Omega(a, b)}$, with $0<a \leq b$, is the (normalised) characteristic function of the ellipse of semi-axes $a$ and $b$. 
The proof we propose in this section uses the explicit expression of the logarithmic potential of $\mu_{a, b}$, namely $-\log |\cdot| * \mu_{a, b}$, which is well-known in the literature, in the context of fluid mechanics. This potential represents the stream function associated to the vorticity corresponding to an elliptic vortex patch (the Kirchhoff ellipse) rotating with constant angular velocity about its centre, and it was computed in order to prove that the Kirchhoff ellipses are V-states of the Euler equations in two dimensions [25,28,30,34].

The explicit expression of the logarithmic potential for any ellipse

$$
\Phi_{a, b}:=-\log |\cdot| * \mu_{a, b}
$$

is well-known (see, e.g., [33, Section 159]) and is given by

$$
\Phi_{a, b}(x)= \begin{cases}-\frac{1}{a b} \frac{b x_{1}^{2}+a x_{2}^{2}}{a+b}-\log \left(\frac{a+b}{2}\right)+\frac{1}{2} & \text { if } x \in \Omega(a, b), \\ H(x) & \text { if } x \in \Omega(a, b)^{c},\end{cases}
$$

where the function $H$ is defined as

$H(x) \equiv H(z)= \begin{cases}-\log |z| & \text { if } a=b, \\ -\frac{1}{c^{2}} \Re\left(z \sqrt{z^{2}+c^{2}}-z^{2}\right)-\log \left|\sqrt{z^{2}+c^{2}}+z\right|+\log 2+\frac{1}{2} & \text { if } a<b .\end{cases}$

We note that $H$ is real-valued, $H(z)=H(\bar{z})$, and that

$$
\nabla H(x) \equiv 2 \bar{\partial} H(\bar{z})=-\frac{1}{\bar{z}}
$$

if $a=b$, whereas

$$
\nabla H(x) \equiv 2 \bar{\partial} H(\bar{z})=-2 h(\bar{z})=-\frac{2}{c^{2}}\left(\sqrt{\bar{z}^{2}+c^{2}}-\bar{z}\right)
$$

for $a<b$.

Note that $\Phi_{a, b}$ is only one part (the radial component) of the convolution potential $W_{\alpha} * \mu_{a, b}$. We now show that the anisotropic part of $W_{\alpha} * \mu_{a, b}$ can be obtained from $\Phi_{a, b}$ by means of an ingenious differentiation. We first write (4.1) explicitly, for $x \in \Omega(a, b)$ and $0<a<b$ :

$$
\Phi_{a, b}(x)=-\frac{1}{\pi a b} \int_{\Omega(a, b)} \log |x-y| d y=-\frac{1}{a b} \frac{b x_{1}^{2}+a x_{2}^{2}}{a+b}-\log \left(\frac{a+b}{2}\right)+\frac{1}{2} .
$$

We perform a change of variables in order to write the integral in the expression above as an integral on the fixed domain $B_{1}(0)$, the unit disc. In terms of the new variables $u=\left(u_{1}, u_{2}\right):=\left(\frac{x_{1}}{a}, \frac{x_{2}}{b}\right), v=\left(v_{1}, v_{2}\right):=\left(\frac{y_{1}}{a}, \frac{y_{2}}{b}\right)$, and the aspect ratio $k:=a / b$, $k \in(0,1)$, the expression in (4.4) becomes

$-\log b-\frac{1}{2 \pi} \int_{B_{1}(0)} \log \left(k^{2}\left(u_{1}-v_{1}\right)^{2}+\left(u_{2}-v_{2}\right)^{2}\right) d v=-\frac{k u_{1}^{2}+u_{2}^{2}}{1+k}-\log \frac{b}{2}-\log (1+k)+\frac{1}{2}$.

By differentiating the previous expression (4.5) with respect to the aspect ratio $k$ we obtain the identity

$$
\frac{1}{\pi} \int_{B_{1}(0)} \frac{k\left(u_{1}-v_{1}\right)^{2}}{k^{2}\left(u_{1}-v_{1}\right)^{2}+\left(u_{2}-v_{2}\right)^{2}} d v=\frac{u_{1}^{2}-u_{2}^{2}}{(1+k)^{2}}+\frac{1}{1+k}, \quad k \in(0,1),
$$


which, expressed in the original variables $x$ and $y$, and $a, b$, becomes

$$
\frac{1}{\pi a b} \int_{\Omega(a, b)} \frac{\left(x_{1}-y_{1}\right)^{2}}{|x-y|^{2}} d y=\frac{a}{a+b}+\frac{b^{2} x_{1}^{2}-a^{2} x_{2}^{2}}{a b(a+b)^{2}} .
$$

Note that the left-hand side of (4.6) is exactly the convolution of the anisotropic term of the potential $W_{\alpha}$ with the measure $\mu_{a, b}$. This allows us to compute the whole convolution potential $W_{\alpha} * \mu_{a, b}$ on $\Omega(a, b)$ :

$$
\begin{aligned}
\left(W_{\alpha} * \mu_{a, b}\right)(x) & =\frac{1}{\pi a b} \int_{\Omega(a, b)}\left(-\log |x-y|+\alpha \frac{\left(x_{1}-y_{1}\right)^{2}}{|x-y|^{2}}\right) d y \\
& =-\frac{1}{a b} \frac{b x_{1}^{2}+a x_{2}^{2}}{a+b}-\log \left(\frac{a+b}{2}\right)+\frac{1}{2}+\alpha \frac{a}{a+b}+\alpha \frac{b^{2} x_{1}^{2}-a^{2} x_{2}^{2}}{a b(a+b)^{2}} \\
& =\frac{-a-b+\alpha b}{a(a+b)^{2}} x_{1}^{2}-\frac{a+b+\alpha a}{b(a+b)^{2}} x_{2}^{2}-\log \left(\frac{a+b}{2}\right)+\frac{1}{2}+\alpha \frac{a}{a+b} .
\end{aligned}
$$

Then we can evaluate the value of the energy $I_{\alpha}$ on ellipses $\mu_{a, b}$, namely

$$
\begin{aligned}
I_{\alpha}\left(\mu_{a, b}\right)= & \frac{1}{2 \pi a b} \int_{\Omega(a, b)}\left(W_{\alpha} * \mu_{a, b}\right)(x) d x+\frac{1}{2 \pi a b} \int_{\Omega(a, b)}\left(x_{1}^{2}+x_{2}^{2}\right) d x \\
= & \frac{1}{2 \pi a b}\left(1+\frac{-a-b+\alpha b}{a(a+b)^{2}}\right) \int_{\Omega(a, b)} x_{1}^{2} d x \\
& +\frac{1}{2 \pi a b}\left(1-\frac{a+b+\alpha a}{b(a+b)^{2}}\right) \int_{\Omega(a, b)} x_{2}^{2} d x \\
& -\frac{1}{2} \log \left(\frac{a+b}{2}\right)+\frac{1}{4}+\frac{\alpha}{2} \frac{a}{a+b},
\end{aligned}
$$

where

$$
\frac{1}{\pi a b} \int_{\Omega(a, b)} x_{1}^{2} d x=\frac{a^{2}}{4} \quad \text { and } \quad \frac{1}{\pi a b} \int_{\Omega(a, b)} x_{2}^{2} d x=\frac{b^{2}}{4} .
$$

In the special case of $a=\sqrt{1-\alpha}$ and $b=\sqrt{1+\alpha}$ we have that

$$
\frac{-a-b+\alpha b}{a(a+b)^{2}}=-\frac{a+b+\alpha a}{b(a+b)^{2}}=-\frac{1}{2},
$$

so that by (4.7) we conclude that $\left(W_{\alpha} * \mu_{\alpha}\right)(x)=-\frac{1}{2}|x|^{2}+C_{\alpha}$ for every $x \in \Omega(\sqrt{1-\alpha}$, $\sqrt{1+\alpha})$ with

$$
\begin{aligned}
C_{\alpha} & =-\log \left(\frac{\sqrt{1-\alpha}+\sqrt{1+\alpha}}{2}\right)+\frac{1}{2}+\alpha \frac{\sqrt{1-\alpha}}{\sqrt{1-\alpha}+\sqrt{1+\alpha}} \\
& =2 I_{\alpha}\left(\mu_{\alpha}\right)-\frac{1}{2} \int_{\mathbb{R}^{2}}|x|^{2} d \mu_{\alpha}(x) .
\end{aligned}
$$

In particular, by (4.8) and (4.9) we obtain the minimum value $I_{\alpha}\left(\mu_{\alpha}\right)$ of the energy $I_{\alpha}$, that is,

$$
I_{\alpha}\left(\mu_{\alpha}\right)=\frac{3}{8}-\frac{1}{2} \log \left(\frac{\sqrt{1-\alpha}+\sqrt{1+\alpha}}{2}\right)+\frac{\alpha}{2} \frac{\sqrt{1-\alpha}}{\sqrt{1-\alpha}+\sqrt{1+\alpha}} .
$$


Remark 4.1 (Computation of $W_{\alpha} * \mu_{a, b}$ ). For completeness, we can compute, for any $b \geq a>0$ and any $x \in \mathbb{R}^{2}$, the value of $\left(W_{\alpha} * \mu_{a, b}\right)(x)$. Equation (4.7) gives

$$
\left(W_{\alpha} * \mu_{a, b}\right)(x)=\frac{-a-b+\alpha b}{a(a+b)^{2}} x_{1}^{2}-\frac{a+b+\alpha a}{b(a+b)^{2}} x_{2}^{2}-\log \left(\frac{a+b}{2}\right)+\frac{1}{2}+\alpha \frac{a}{a+b}
$$

for any $x \in \Omega(a, b)$. Outside the ellipse, it is again convenient to pass to complex variables. Integrating (3.7) with respect to $\bar{z}$, and recalling (4.2) and (4.3), we can show that

$$
\left(W_{\alpha} * \mu_{a, b}\right)(z)=H(z)+\alpha \Re\left(h(z) \bar{z}-a b h(\bar{z})^{2}-\lambda h(\bar{z}) \bar{z}\right)+\alpha \frac{a}{a+b}
$$

for any $z \in \Omega(a, b)^{c}$.

4.2. More general anisotropy. Now we briefly discuss the case of a more general anisotropy of the type

$$
V_{\alpha, \beta, \gamma}\left(x_{1}, x_{2}\right):=\frac{\alpha x_{1}^{2}+\beta x_{2}^{2}+\gamma x_{1} x_{2}}{x_{1}^{2}+x_{2}^{2}},
$$

where $\alpha, \beta, \gamma \in \mathbb{R}$. Let $W_{\alpha}^{\gamma}$ be the kernel defined as

$$
W_{\alpha, \beta, \gamma}\left(x_{1}, x_{2}\right):=-\log |x|+\frac{\alpha x_{1}^{2}+\beta x_{2}^{2}+\gamma x_{1} x_{2}}{x_{1}^{2}+x_{2}^{2}},
$$

and let $I_{\alpha, \beta, \gamma}$ be the corresponding energy, defined on probability measures $\mu \in \mathcal{P}\left(\mathbb{R}^{2}\right)$ as

$$
I_{\alpha, \beta, \gamma}(\mu):=\frac{1}{2} \iint_{\mathbb{R}^{2} \times \mathbb{R}^{2}} W_{\alpha, \beta, \gamma}(x-y) d \mu(x) d \mu(y)+\frac{1}{2} \int_{\mathbb{R}^{2}}|x|^{2} d \mu(x) .
$$

If $\gamma=0$, the anisotropy (4.10) can be written as

$$
V_{\alpha, \beta, 0}\left(x_{1}, x_{2}\right)=(\alpha-\beta) \frac{x_{1}^{2}}{x_{1}^{2}+x_{2}^{2}}+\beta,
$$

so that the study of minimisers of $I_{\alpha, \beta, 0}$ is covered by the previous analysis.

Assume $\gamma \neq 0$. Consider the rotation in the plane defined by

$$
y=\frac{1}{\sqrt{a^{2}+\gamma^{2}}}\left(\begin{array}{cc}
-a & \gamma \\
-\gamma & -a
\end{array}\right) x
$$

where $a:=\beta-\alpha-\sqrt{(\beta-\alpha)^{2}+\gamma^{2}}$. Setting $b:=\sqrt{(\beta-\alpha)^{2}+\gamma^{2}}$, a simple computation shows that

$$
V_{\alpha, \beta, \gamma}\left(x_{1}, x_{2}\right)=b \frac{y_{1}^{2}}{y_{1}^{2}+y_{2}^{2}}+\beta-\frac{b \gamma^{2}}{a^{2}+\gamma^{2}},
$$

so that, up to this change of variables, the study of the minimality of $I_{\alpha, \beta, \gamma}$ reduces again to the original case. In particular, for $b<1$ the minimiser is an ellipse with major axis 
along the line $y_{1}=0$, that is, $-a x_{1}+\gamma x_{2}=0$, while for $b \geq 1$ the minimiser is the semi-circle law on that line.

The two orthogonal lines $y_{1}=0$ and $y_{2}=0$ are the zero set of the anisotropic force $F_{\alpha, \beta, \gamma}$, given by

$$
F_{\alpha, \beta, \gamma}(x)=-\nabla V_{\alpha, \beta, \gamma}(x)=\frac{\gamma x_{1}^{2}-\gamma x_{2}^{2}+2(\beta-\alpha) x_{1} x_{2}}{\left(x_{1}^{2}+x_{2}^{2}\right)^{2}} x^{\perp},
$$

where $x^{\perp}=\left(x_{2},-x_{1}\right)$. The force $F_{\alpha, \beta, \gamma}$ is perpendicular to the radial direction, and it is indeed zero only when

$$
x_{2}=\frac{1}{\gamma}\left(\beta-\alpha \pm \sqrt{(\beta-\alpha)^{2}+\gamma^{2}}\right) x_{1},
$$

which correspond to $y_{1}=0$ and $y_{2}=0$. Looking at the sign of the force in (4.11) it is clear that $F_{\alpha, \beta, \gamma}$ points towards the line $y_{1}=0$.

Acknowledgements. JAC was partially supported by the Royal Society via a Wolfson Research Merit Award and by the EPSRC under the Grant EP/P031587/1. MGM and LR are partly supported by GNAMPA-INdAM. MGM acknowledges support by the European Research Council under Grant No. 290888. LR acknowledges support by the Università di Trieste through FRA 2016. LS acknowledges support by the EPSRC under the Grant EP/N035631/1. JM and JV acknowledge support by the Spanish projects MTM2013-44699 (MINECO) and MTM2016-75390 (MINECO), 2014SGR75 (Generalitat de Catalunya) and FP7-607647 (European Union).

Open Access This article is distributed under the terms of the Creative Commons Attribution 4.0 International License (http://creativecommons.org/licenses/by/4.0/), which permits unrestricted use, distribution, and reproduction in any medium, provided you give appropriate credit to the original author(s) and the source, provide a link to the Creative Commons license, and indicate if changes were made.

Publisher's Note Springer Nature remains neutral with regard to jurisdictional claims in published maps and institutional affiliations.

\section{References}

1. Albi, G., Balagué, D., Carrillo, J.A., von Brecht, J.: Stability analysis of flock and mill rings for second order models in swarming. SIAM J. Appl. Math. 74, 794-818 (2014)

2. Ambrosio, L., Gigli, N., Savaré, G.: Gradient Flows in Metric Spaces and in the Space of Probability Measures. Birkhäuser, Basel (2005)

3. Balagué, D., Carrillo, J.A., Laurent, T., Raoul, G.: Dimensionality of local minimizers of the interaction energy. Arch. Ration. Mech. Anal. 209, 1055-1088 (2013)

4. Balogh, F., Merzi, D.: Equilibrium measures for a class of potentials with discrete rotational symmetries. Constr. Approx. 42, 399-424 (2015)

5. Bertozzi, A.L., Kolokolnikov, T., Sun, H., Uminsky, D., von Brecht, J.: Ring patterns and their bifurcations in a nonlocal model of biological swarms. Commun. Math. Sci. 13, 955-985 (2015)

6. Bertozzi, A.L., Laurent, T., Léger, F.: Aggregation and spreading via the Newtonian potential: the dynamics of patch solutions. Math. Models Methods Appl. Sci. 22, 1140005, 39pp (2012)

7. Blanchet, A., Carlier, G.: From Nash to Cournot-Nash equilibria via the Monge-Kantorovich problem. Philos. Trans. R. Soc. Lond. Ser. A Math. Phys. Eng. Sci. 372, 20130398, 11pp (2014)

8. Bleher, P.M., Delvaux, S., Kuijlaars, A.B.J.: Random matrix model with external source and a constrained vector equilibrium problem. Comm. Pure Appl. Math. 64, 116-160 (2011)

9. Bleher, P.M., Kuijlaars, A.B.J.: Orthogonal polynomials in the normal matrix model with a cubic potential. Adv. Math. 230, 1272-1321 (2012)

10. Brézis, H., Kinderlehrer, D.: The smoothness of solutions to nonlinear variational inequalities. Indiana Univ. Math. J. 23, 831-844 (1974)

11. Burbea, J.: Motions of vortex patches. Lett. Math. Phys. 6, 1-16 (1982)

12. Caffarelli, L.A.: The obstacle problem revisited. J. Fourier Anal. Appl. 4, 383-402 (1998) 
13. Caffarelli, L.A., Friedman, A.: A singular perturbation problem for semiconductors. Boll. Un. Mat. Ital. B (7) 1, 409-421 (1987)

14. Caffarelli, L.A., Vázquez, J.L.: Nonlinear porous medium flow with fractional potential pressure. Arch. Ration. Mech. Anal. 202, 537-565 (2011)

15. Caffarelli, L.A., Vázquez, J.L.: Asymptotic behaviour of a porous medium equation with fractional diffusion. Discrete Contin. Dyn. Syst. 29, 1393-1404 (2011)

16. Cañizo, J.A., Carrillo, J.A., Patacchini, F.S.: Existence of compactly supported global minimisers for the interaction energy. Arch. Ration. Mech. Anal. 217, 1197-1217 (2015)

17. Carrillo, J.A., Castorina, D., Volzone, B.: Ground states for diffusion dominated free energies with logarithmic interaction. SIAM J. Math. Anal. 47, 1-25 (2015)

18. Carrillo, J.A., Delgadino, M.G., Mellet, A.: Regularity of local minimizers of the interaction energy via obstacle problems. Commun. Math. Phys. 343, 747-781 (2016)

19. Carrillo, J.A., Figalli, A., Patacchini, F.S.: Geometry of minimizers for the interaction energy with mildly repulsive potentials. Ann. Inst. H. Poincaré Anal. Nonlinear 34, 1299-1308 (2017)

20. Carrillo, J.A., Huang, Y.: Explicit equilibrium solutions for the aggregation equation with power-law potentials. Kinet. Relat. Models. 10, 171-192 (2017)

21. Carrillo, J.A., McCann, R.J., Villani, C.: Kinetic equilibration rates for granular media and related equations: entropy dissipation and mass transportation estimates. Rev. Mat. Iberoam. 19, 971-1018 (2003)

22. Carrillo, J.A., Vázquez, J.L.: Some free boundary problems involving non-local diffusion and aggregation. Philos. Trans. R. Soc. Lond. Ser. A Math. Phys. Eng. Sci. 373, 20140275, 16pp (2015)

23. Dal Maso, G.: An Introduction to $\Gamma$-Convergence. Birkhäuser, Boston (1993)

24. D’Orsogna, M.R., Chuang, Y.-L., Bertozzi, A.L., Chayes, L.S.: Self-propelled particles with soft-core interactions: patterns, stability, and collapse. Phys. Rev. Lett. 96, 104302, 4pp (2006)

25. Flierl, G.R., Polvani, L.M: Generalized Kirchhoff vortices. Phys. Fluids 29, 2376-2379 (1986)

26. Frostman, O.: Potentiel d'équilibre et capacité des ensembles avec quelques applications à la théorie des fonctions. Meddel. Lunds Univ. Mat. Sem. 3, 1-118 (1935)

27. Geers, M.G.D., Peerlings, R.H.J., Peletier, M.A., Scardia, L.: Asymptotic behaviour of a pile-up of infinite walls of edge dislocations. Arch. Ration. Mech. Anal. 209, 495-539 (2013)

28. Hmidi, T., Mateu, J., Verdera, J.: On rotating doubly connected vortices. J. Differ. Equ. 258, 13951429 (2015)

29. Holm, D.D., Putkaradze, V.: Formation of clumps and patches in self-aggregation of finite-size particles. Phys. D 220, 183-196 (2006)

30. Kirchhoff, G.: Vorlesungen über mathematische Physik. Teubner, Leipzig (1874)

31. Kolokolnikov, T., Sun, H., Uminsky, D., Bertozzi, A.L.: Stability of ring patterns arising from twodimensional particle interactions. Phys. Rev. E 84, 015203, 4pp (2011)

32. Kuijlaars, A.B.J., Dragnev, P.D.: Equilibrium problems associated with fast decreasing polynomials. Proc. Am. Math. Soc. 127, 1065-1074 (1999)

33. Lamb, H.: Hydrodynamics. Cambridge University Press, Cambridge (1932)

34. Mitchell, T.B., Rossi, L.F.: The evolution of Kirchhoff elliptic vortices. Phys. Fluids 20, 054103, 12pp (2008)

35. Mogilner, A., Edelstein-Keshet, L.: A non-local model for a swarm. J. Math. Biol. 38, 534-570 (1999)

36. Mora, M.G., Peletier, M., Scardia, L.: Convergence of interaction-driven evolutions of dislocations with Wasserstein dissipation and slip-plane confinement. SIAM J. Math. Anal. 49, 4149-4205 (2017)

37. Mora, M.G., Rondi, L., Scardia, L.: The equilibrium measure for a nonlocal dislocation energy. Commun. Pure Appl. Math. 72, 136-158 (2019)

38. Otto, F.: The geometry of dissipative evolution equations: the porous medium equation. Commun. Partial Differ. Equ. 26, 101-174 (2001)

39. Saff, E.B., Totik, V.: Logarithmic Potentials with External Fields. Springer, Berlin (1997)

40. Serfaty, S., Vázquez, J.L.: A mean field equation as limit of nonlinear diffusions with fractional Laplacian operators. Calc. Var. Partial Differ. Equ. 49, 1091-1120 (2014)

41. Simione, R., Slepčev, D., Topaloglu, I.: Existence of ground states of nonlocal-interaction energies. J. Stat. Phys. 159, 972-986 (2015)

42. Stein, E.M.: Singular Integrals and Differentiability Properties of Functions. Princeton University Press, Princeton (1970)

43. Topaz, C.M., Bertozzi, A.L., Lewis, M.A.: A nonlocal continuum model for biological aggregation. Bull. Math. Biol. 68, 1601-1623 (2006)

44. Toscani, G.: One-dimensional kinetic models of granular flows. M2AN Math. Model. Numer. Anal. 34, 1277-1291 (2000)

45. Villani, C.: Topics in Optimal Transportation. American Mathematical Society, Providence (2003) 